\title{
Heiliger Wald - oder der Park der Monster in Bomarzo/Italien
}

\author{
Hilke Steinecke \& Peter Schubert
}

\begin{abstract}
The park Sacro Bosco or Parco dei Mostri is located about $80 \mathrm{~km}$ north of Rome at Bomarzo. It was commissioned by Vicino Orsini in the 16th century. Huge stone-carved sculptures await the visitor in the forest-like park. It is above all beings from the Greek and Roman mythology such as the hellhound Cerberus or the winged horse Pegasus. Outside Italy's borders the huge head of OrCus with its walk-in mouth, the hellmouth, is famous.
\end{abstract}

\section{Zusammenfassung}

Der Park Sacro Bosco oder Parco dei Mostri liegt etwa 80 km nördlich von Rom bei Bomarzo. Er wurde im 16. Jahrhundert von Vicino Orsini in Auftrag gegeben. Riesige aus Stein gehauene Skulpturen erwarten den Besucher in dem waldartigen Park. Es sind vor allem Wesen aus der griechischen und römischen Mythologe wie der Höllenhund Cerberus oder das geflügelte Pferd Pegasus. Über die Grenzen Italiens hinaus bekannt ist der riesige Kopf von Orcus mit seinem begehbaren Maul, dem Höllenschlund.

\section{Einführung}

Sacro Bosco (Heiliger Wald) ist wohl einer der außergewöhnlichsten historischen Parks Italiens. Der Park stammt aus dem 16. Jahrhundert. Hier werden viele Gestalten aus der griechischen und römischen Mythologie und andere Fabelwesen in Szene gesetzt. Wegen seiner riesigen, in Stein gemeißelten Ungeheuer und Fabelwesen wird er in neuerer Zeit, vermutlich aus Gründen der Ver- marktung, auch Parco dei Mostri (Park der Monster) genannt. Der Park befindet sich etwa $80 \mathrm{~km}$ nördlich von Rom unterhalb der auf einem Felsen errichteten Stadt Bomarzo im Norden der Region Latium. Er liegt verwunschen in einem Waldstück, offenbart viele Geheimnisse und erfreut mit seinen fantasieanregenden Skulpturen zahlreiche Besucher. Darunter sind nicht nur reichlich auswärtigen Touristen, sondern auch viele Familien

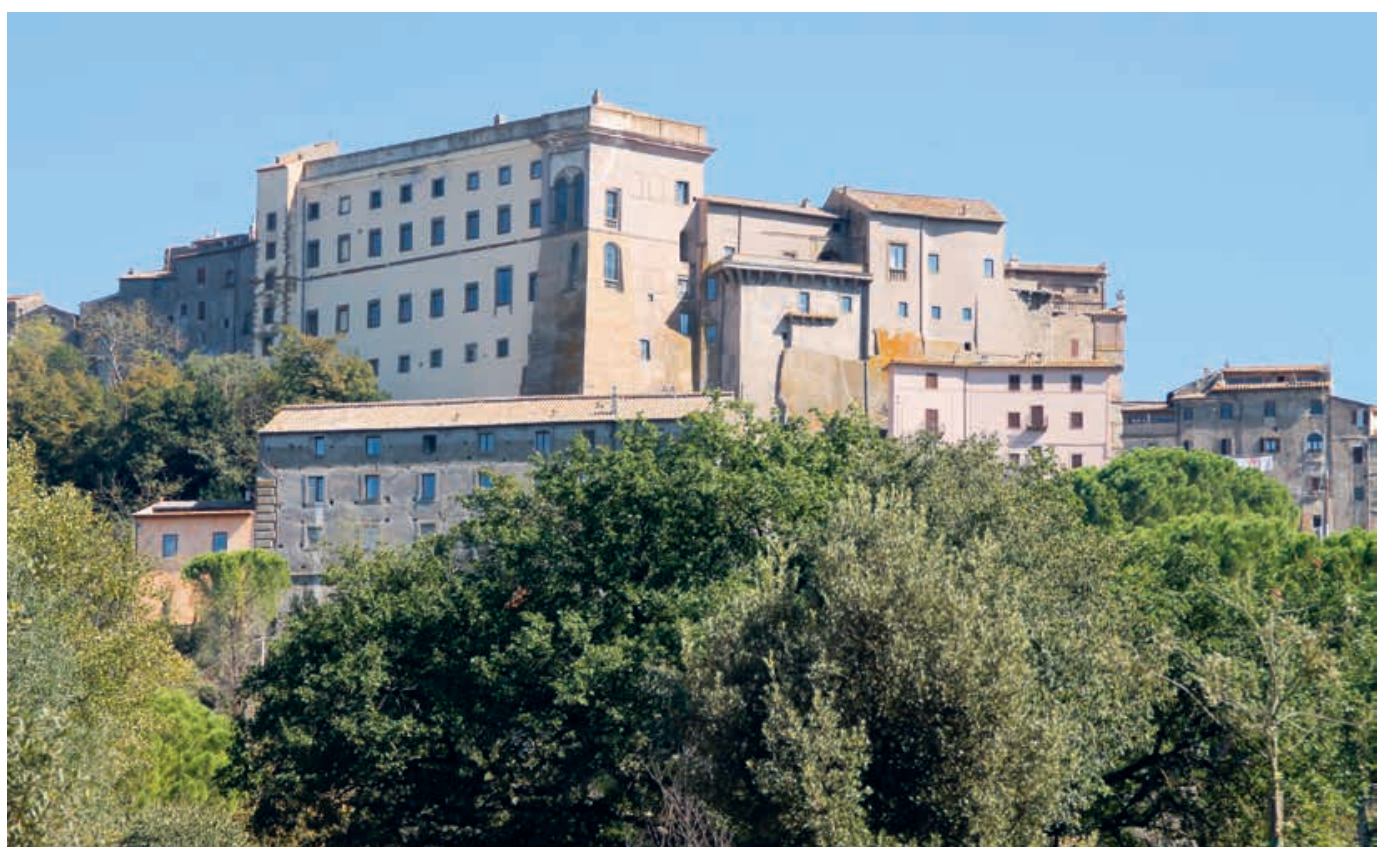

Abb. 1: Palazzo Orsini in Bomarzo. (Foto: P. Schubert) 


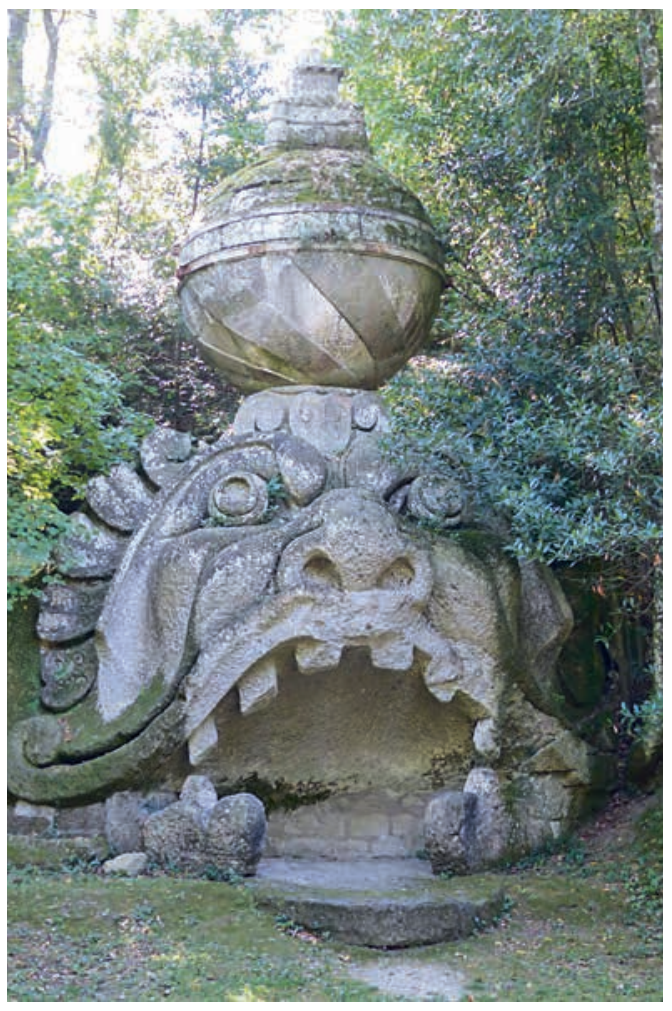

Abb. 2: Glaukos mit geöffnetem Maul. (Foto: H. Steinecke)

mit Kindern aus der Region. Der Einzugsbereich für einen Tagesauflug reicht bis nach Rom.

\section{Geschichte}

Im Palazzo Orsini, der in dem über dem Park gelegenen alten Ort Bomarzo liegt, lebte VICINo Orsini (1523-1585; 1542 Übernahme der Grafschaft Bomarzo von seinem Vater). Er war der letzte Feudalherr von Bomarzo, zusammen mit seiner Gattin GiUlia Farnese. Die Farnese gehörten seit dem 11. Jahrhundert zu den bedeutendsten italienischen Adelsfamilien, und noch heute bezeugen einige prachtvolle Paläste in der Provinz Viterbo die einstige Macht und den Reichtum der Familie. Aus dieser Adelsdynastie entstammte auch Allessandro Farnese, der 1534 zum Papst PAul III. gewählt wurde. Nachdem Vicino Orsini im Jahr 1557 Zeuge einer Schlacht geworden war, die mit einem Massaker an der Zivilbevölkerung endete, gab er seine politische und militärische Karriere auf. Der humanistisch Gebildete widmete sich fortan

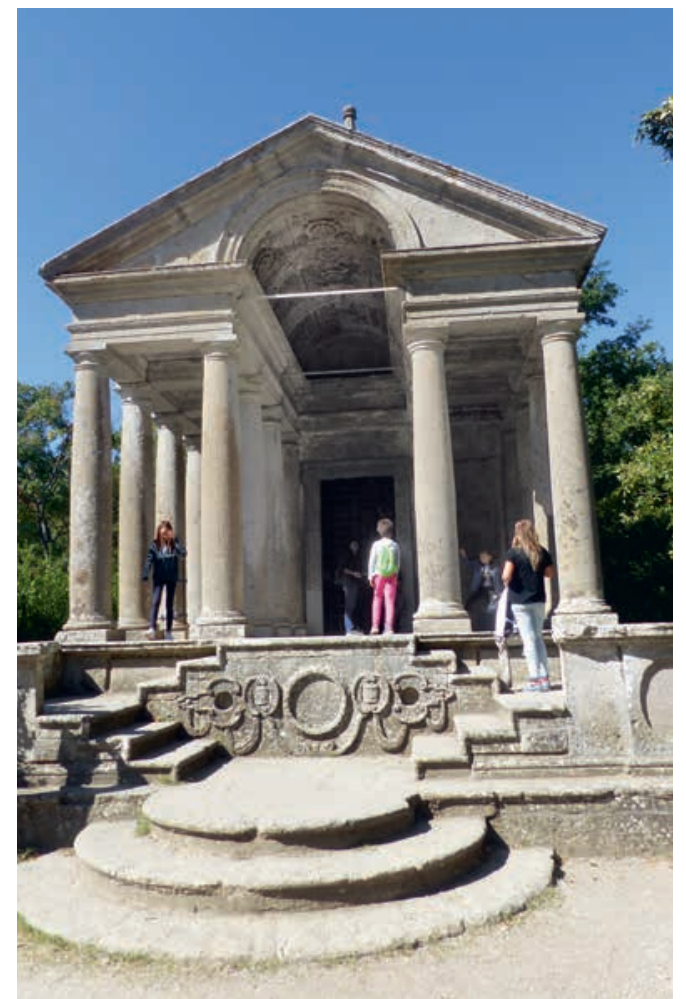

Abb. 3: Das Mausoleum. (Foto: H. Steinecke)

vor allem der Philosophie, zusammen mit seiner Ehefrau als Mäzenin.

Giulia Farnese inspirierte und unterstützte ihren Gatten, Sacro Bosco, den heilig-geheimnisvollen und märchenhaften Waldpark, zu schaffen. DA Vicino und Givlia über genügend finanzielle Mittel verfügten, konnte dieser Traum umgesetzt werden. Über drei Jahrzehnte lang, von 1552 bis zu seinem Tod im Jahre 1585, ließ Vicino den Park von den damals renommiertesten Architekten und Gartengestaltern Pirro Ligorio und Giacomo Barozzi da Vignola anlegen. Orsinis Philosophie bestand darin, eine phantastische, groteske und obszöne Welt zu inszenieren. Die Normalität sollte dabei aus den Fugen geraten, sexuelle Tabus gebrochen und ein Gegenpol zur starren katholischen Denkweise der katholischen Kirche gesetzt werden. Jahr für Jahr kamen neue Skulpturen hinzu. Sie wurden aus dem lokal anstehenden basaltischen Tuffgestein (Peperin) gehauen. Dieser ist relativ leicht zu bearbeiten und 


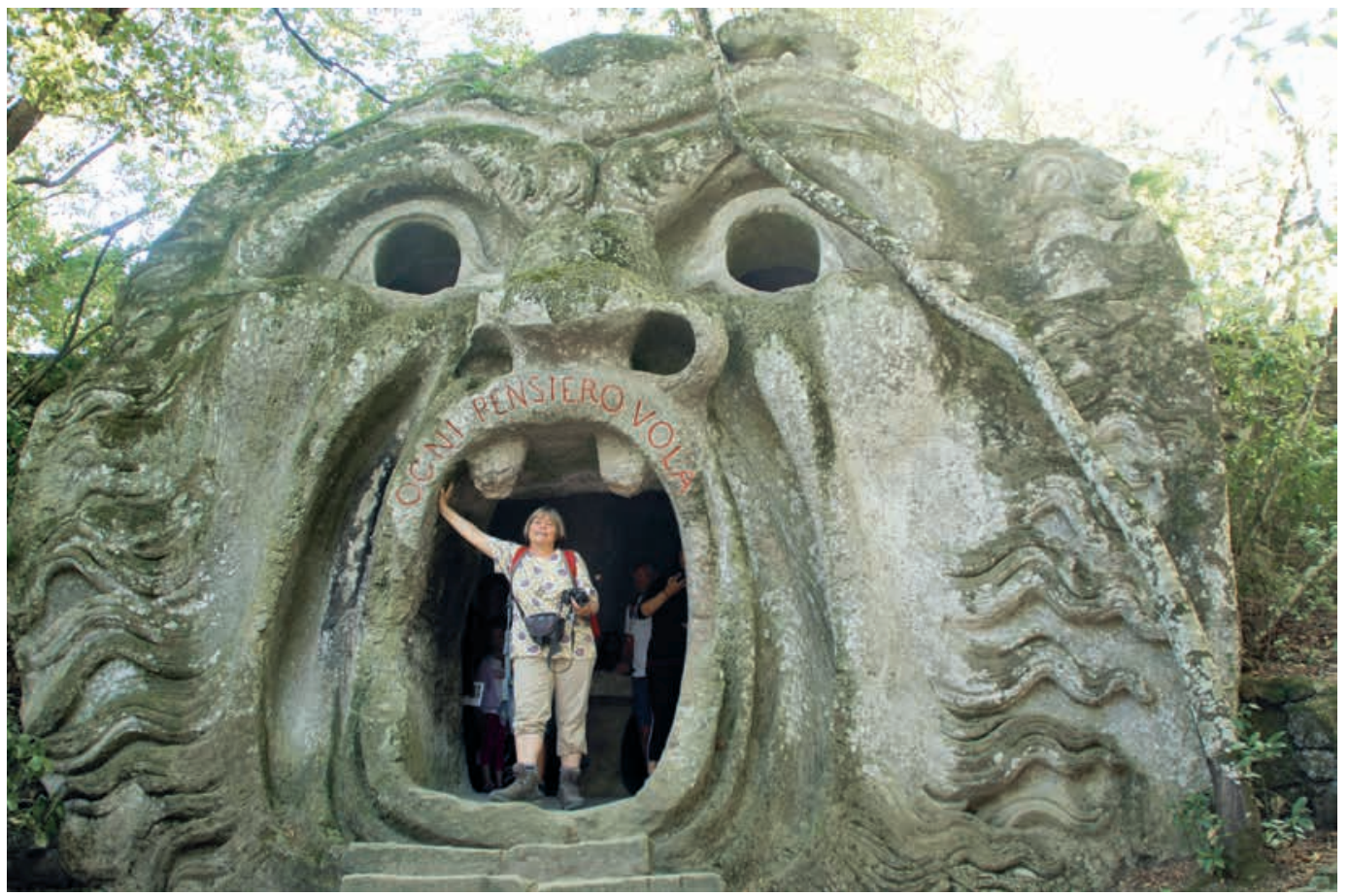

Abb. 4. Der berühmte Höllenschlund des Monsters Orcus. (Foto: P. SCHubert)

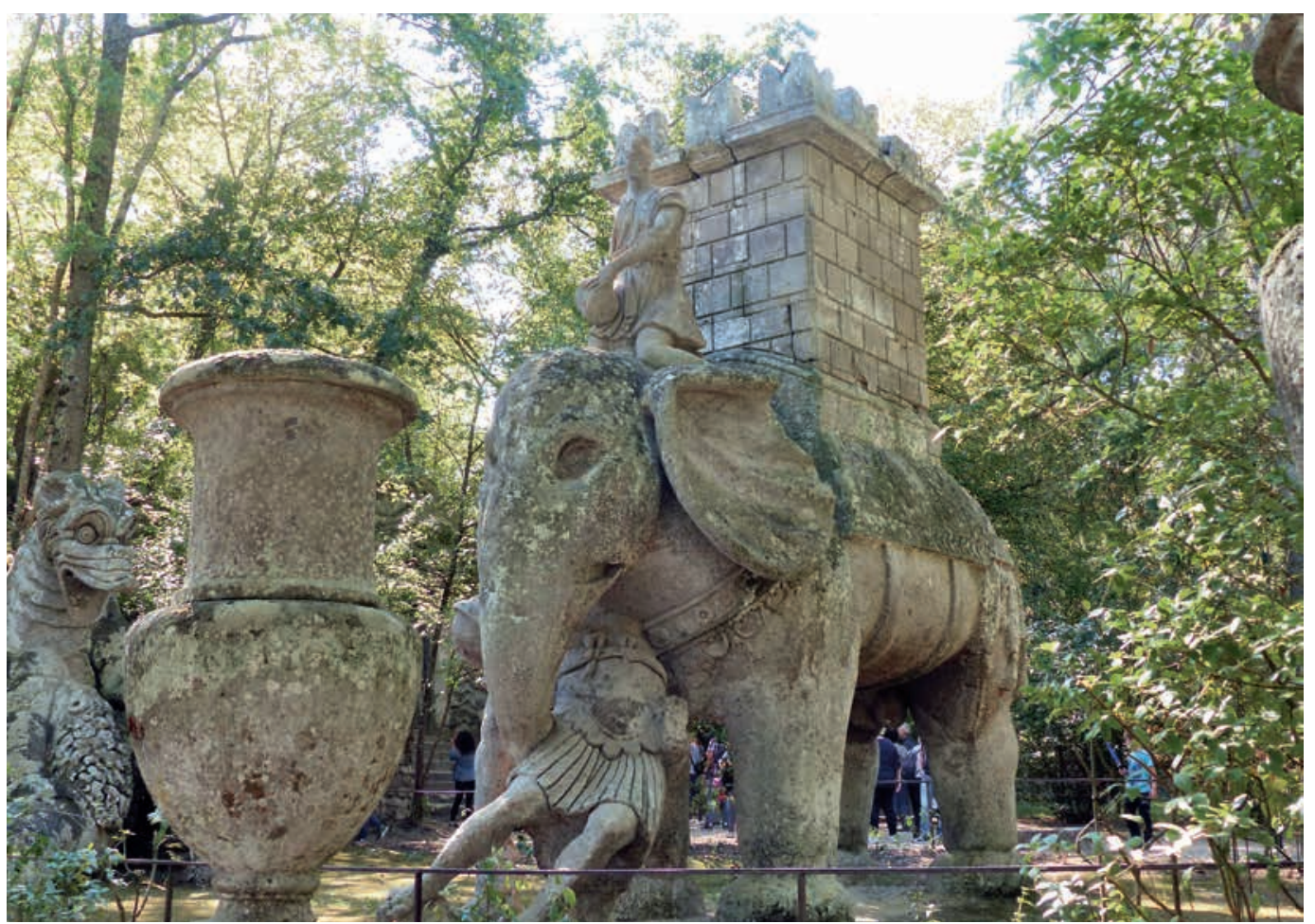

Abb. 5: Ein überlebensgroßer steinerner Elefant. (Foto: H. Steinecke) 
kommt vor allem im Albanergebirge bei Rom vor. Schon im Altertum wurde Peperin dort traditionell als Baumaterial (Lapis albanus) benutzt.

Givlia konnte nur die Anfänge des Parks miterleben, denn sie verstarb bereits 1564. Ihr Gatte widmete aber ihr den Sacro Bosco, den er bewusst nicht als Park bezeichnet hatte und den er zeitlebens erweiterte. Während er vor Giulias Tod vor allem die griechische und römische Sagenwelt thematisierte, widmete er sich danach besonders auch den Themen Tod und Unterwelt.

Im Heiligen Wald wurden Stilelemente des Manierismus eingesetzt. Dazu gehören die Auflösung und Verzerrung der starren Formen der Renaissance, die Verwendung grotesker Ornamentik und unüblicher Proportionen. So stehen auch die einzelnen Skulpturen von Sacro Bosco nicht in einem größeren gestalterischen Zusammenhang und es gibt anders als in vielen Renaissancegärten der damaligen Zeit keine auffälligen Sichtachsen.

Nach Orsinis Tod 1585 interessierte sich niemand mehr für den Park. Er verwilderte, die Skulpturen wurden völlig überwachsen und fielen teilweise sogar um, so dass der Park in Vergessenheit geriet. Nur wenige Intellektuelle statteten ihm später noch einen Besuch ab, darunter JoHanN Wolfgang v. Goethe auf seiner Italienreise.

Erst in der Mitte des 20. Jahrhunderts wurde der Park wiederentdeckt und, vielleicht ähnlich wie „The Lost Gardens of Heligan“ in Cornwall, aus seinem Dornröschenschlaf aufgeweckt. Im Jahr 1938 war der Künstler Salvador Dalí ein prominenter Besucher des „neuen“ alten Sacro Bosco. Er war von dem Park so begeistert und inspiriert, dass er seine Eindrücke dieses außergewöhnlichen Parks in seinem Gemälde „Die Versuchung des heiligen Antonius" einfließen ließ.

Giancarlo und Tina Severi Bettini erwarben im Jahr 1954 Sacro Bosco. Indem sie ihn restaurierten, erweckten sie ihn zu neuem Leben. Gartenwissenschaftler und Kunsthistoriker wurden daraufhin auf ihn aufmerksam. Bald wurde Sacro Bosco auch außerhalb der Grenzen Italiens

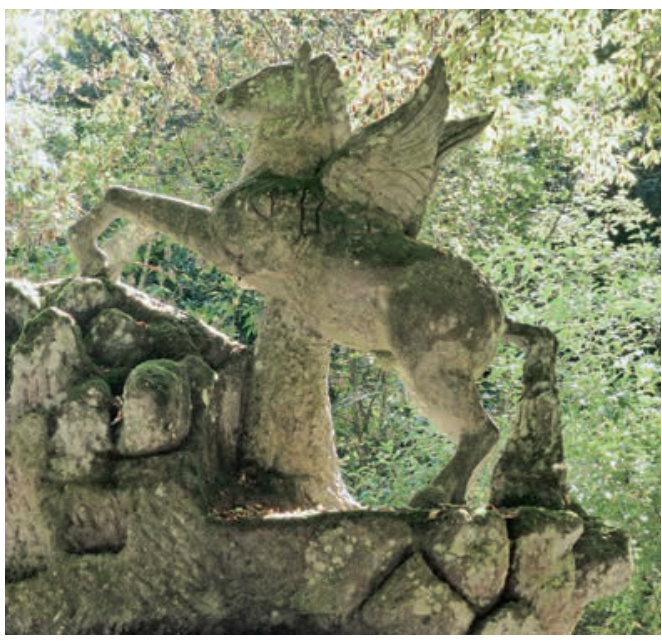

Abb. 6: Das geflügelte Pferd Pegasus. (Foto: H. Steinecke)

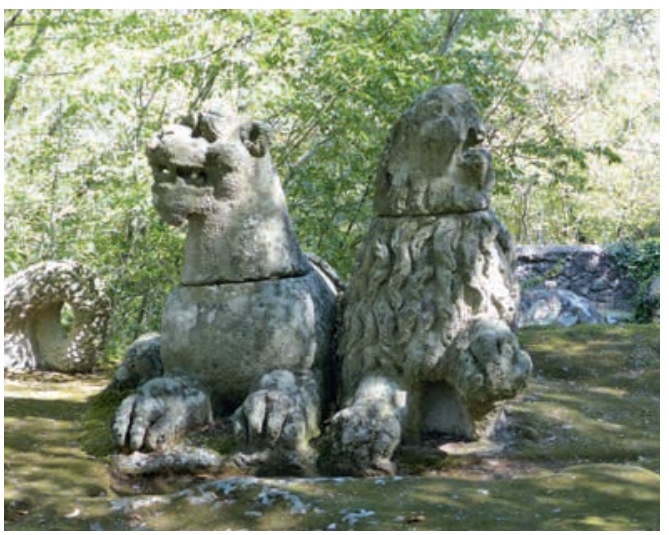

Abb. 7: Der zweiköpfige Höllenhund Cerberus.

(Foto: P. Schubert)

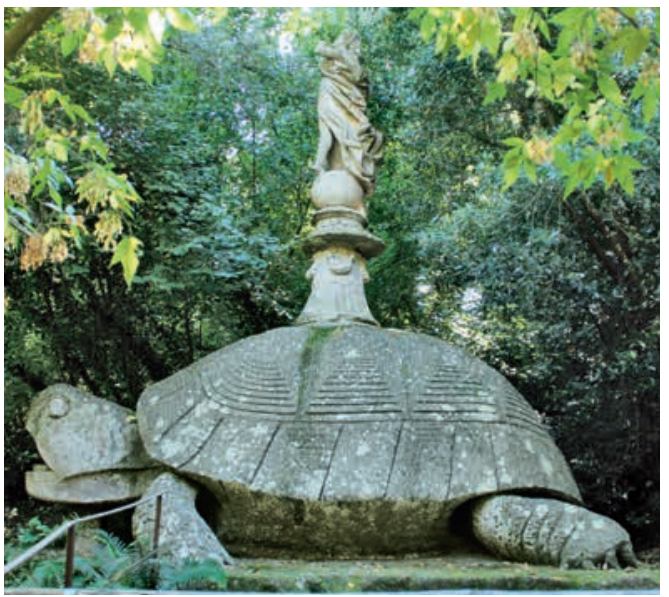

Abb. 8: Eine Riesen-Schildkröte. (Foto: P. Schubert) 


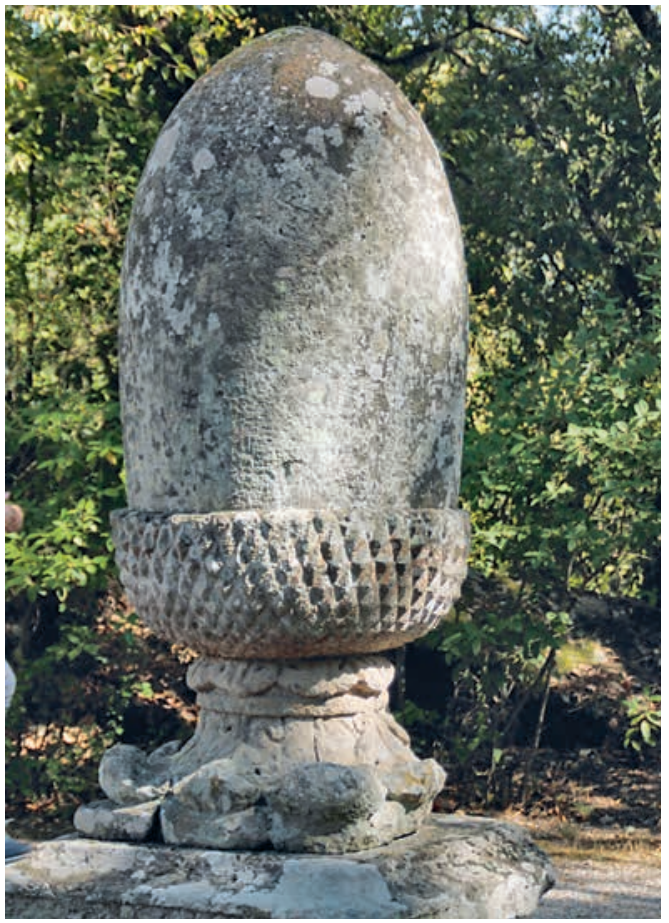

Abb. 9: Eine steinerne Riesen-Eichel. (Foto: P. Schubert)

bekannt. Nach dem Tod des Restauratoren-Paares Bettini wurde der Park eine wichtige Einnahmequelle für die Stadt Bomarzo, die sich heute um ihn kümmert und eine intensivere Werbung und Vermarktung vorantreibt.

\section{Eingangsbereich}

Von Bomarzo kommend zeugt ein großer Parkplatz bereits für die hohe Besucherzahl. Der Eintritt ist mit 10 Euro relativ hoch, zu bedenken ist aber, dass die Skulpturen kontinuierlich instand gehalten und restauriert werden müssen. Im Eingangsbereich gibt es Kinderspielplätze, Schnell-Restaurant und einen Picknickbereich. Ein Weg durch einen Obstgarten führt schließlich zum eigentlichen Portal in diese magische Gartenwelt. Anhand eines Planes können sich die Besucher orientieren. Gleich hinter dem Eingang empfängt ein großes steinernes Ungeheuer mit einem großen, aufgerissenen Maul die Gäste. Es stellt Glaukos dar, einen Meeresgott, in den sich ein Fischer nach dem Verzehr eines Wunderkrauts verwandelte. Glaukos ist eine der vielen Figuren des Parks, die der griechischen oder römischen

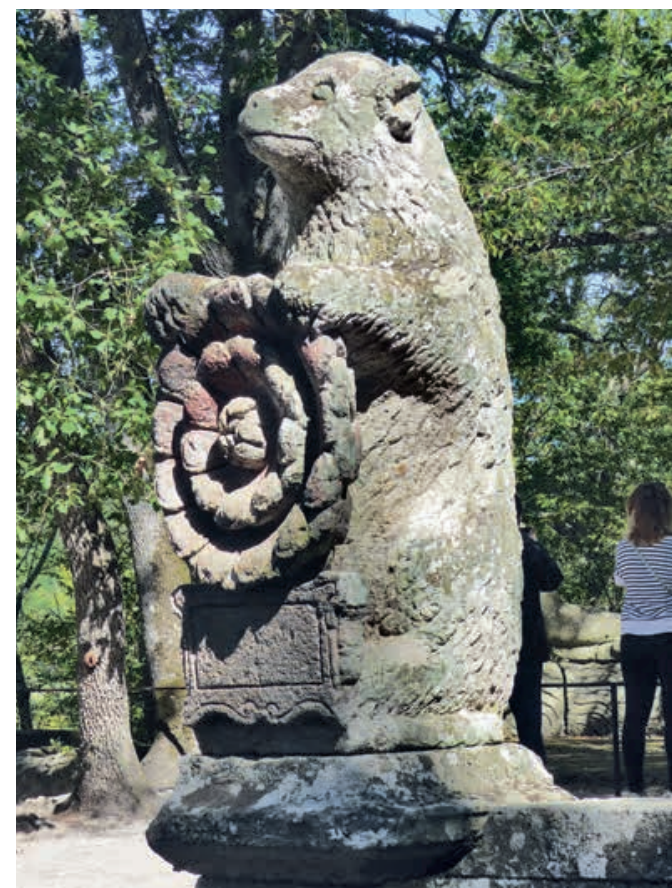

Abb. 10: Der Bär ist eine Anspielung auf den Familiennamen der Orsinis. (Foto: H. Steinecke)

Mythologie entstammen. Weiterhin gibt es hier Anspielungen auf die Literatur der Renaissance. An verschiedenen Stellen sind in den Stein Inschriften gemeißelt. Experten haben versucht, diese zu interpretieren oder ein Konzept zu erkennen; es konnte aber bisher keine klare Antwort dazu gegeben werden. Es gibt Stimmen, die meinen, dass der kunsthistorisch und literarisch gebildete Orsini viele zusammenhanglose Anspielungen auf Kunst und Literatur verwendet hat, um seine Gäste bewusst zu verwirren und zum Nachdenken anzuregen. In einer Inschrift des Parks heißt es in deutscher Übersetzung: „Der Du hier eintrittst und versuchst, alles von Anfang bis Ende zu verstehen, sage, ob so viele Wunder geschaffen wurden, um den Fehler der Kunst zu begehen."

Ganz in der Nähe von Glaukos steht auf einem Plateau ein Tempel. Es ist nicht sicher, ob dieser als Mausoleum für Giulia FARnese errichtet wurde, auf jeden Fall sind hier die BETTINIs begraben. 


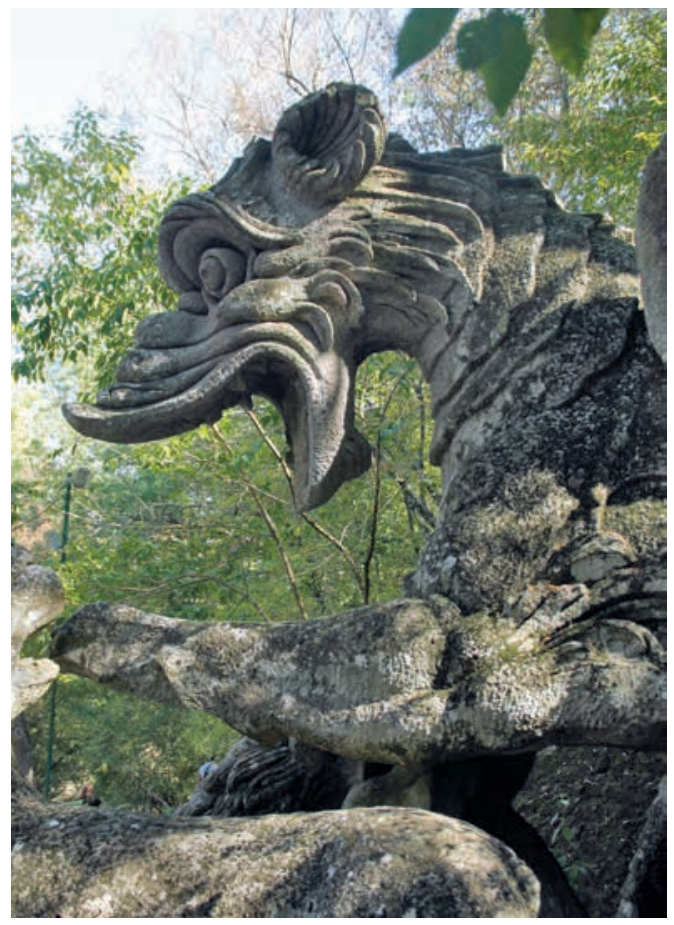

Abb. 11: Ein wilder Drache. (Foto: P. Schubert)

\section{Monster und andere Monumentalfiguren}

Das wohl bekannteste Motiv aus dem Monsterpark ist Orcus, nach römischer Mythologie der Gott der Unterwelt. Eine Treppe führt zu dem riesigen dunklen, geöffneten und begehbaren Maul (Bocca Nera) des Monsters. Auf seiner Oberlippe ist folgende Inschrift zu lesen: „Ogni pensiero vola“ („Jeder Gedanke fliegt“). Im Innern des Höllenschlunds befindet sich sogar eine Sitzgruppe. Früher traf sich hier der Adelige mit seinen intellektuellen Freunden zu ausgiebigen Festgelagen. Der Höllenschlund wird jeden Tag unzählige Mal fotografiert. Eine Familie nach der anderen stellt sich ins Maul und lässt sich am Abgrund zur Hölle ablichten, wobei das trotz des großen Andrangs erstaunlich ruhig abläuft und sich niemand vordrängelt.

Sehr reizvoll sind auch eine riesige steinerne griechische Vase sowie eine sogenannte etruskische Bank. Eine Terrasse wird eindrucksvoll von mannsgroßen Eicheln und Kiefernzapfen flankiert. Weiterhin kann man Neptun, einer schlafenden Frau, einem wilden Löwen und einem Drachen

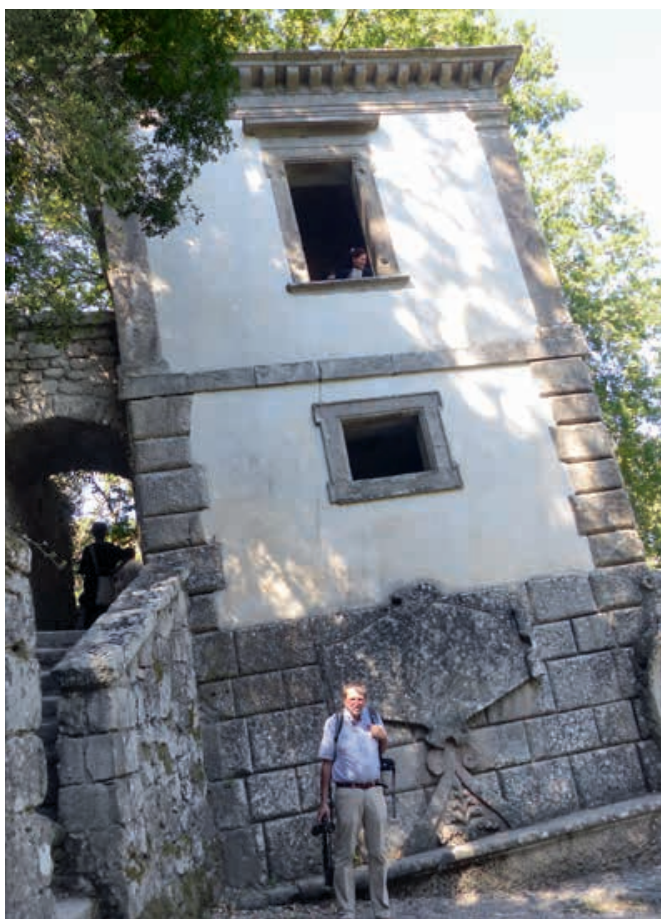

Abb. 12: Casa Pendente. (Foto: H. Steinecke)

begegnen. Einen besonderen Charme haben die Figuren auch deshalb, weil sie mitunter von Moosen überwachsen sind. Vermutlich eine Anspielung auf Hannibals Überquerung der Alpen ist ein überlebensgroßer Elefant mit Reiter und Turm als Gepäck auf dem Rücken. Mit seinem Rüssel umschlingt er einen römischen Legionär.

Weitere Wesen sind zwei große Bären: der Bär ist schließlich Symboltier der Familie OrsINI (= kleiner Bär). Es gibt hier Sphinxe, Sirenen, kämpfende Giganten, das geflügelte Pferd PEGAsus, Ceres und einen zweiköpfigen Höllenhund Cerberus. Besonders geheimnisvoll wirkt eine riesige Schildkröte, die oberhalb des kleinen Tälchens steht, das mit einem Bachlauf das Gelände durchzieht. Auf ihrem Panzer trägt sie eine Frau, deren Deutung unklar ist. Und zwischen all diesen schaurig-schönen Fabelwesen hat man es sich gutgehen lassen. Ein steinernes Wasserbecken diente früher vermutlich als Badewanne.

Auf keinen Fall versäumen sollte man den Besuch des schiefen Hauses (Casa Pendente). Das 


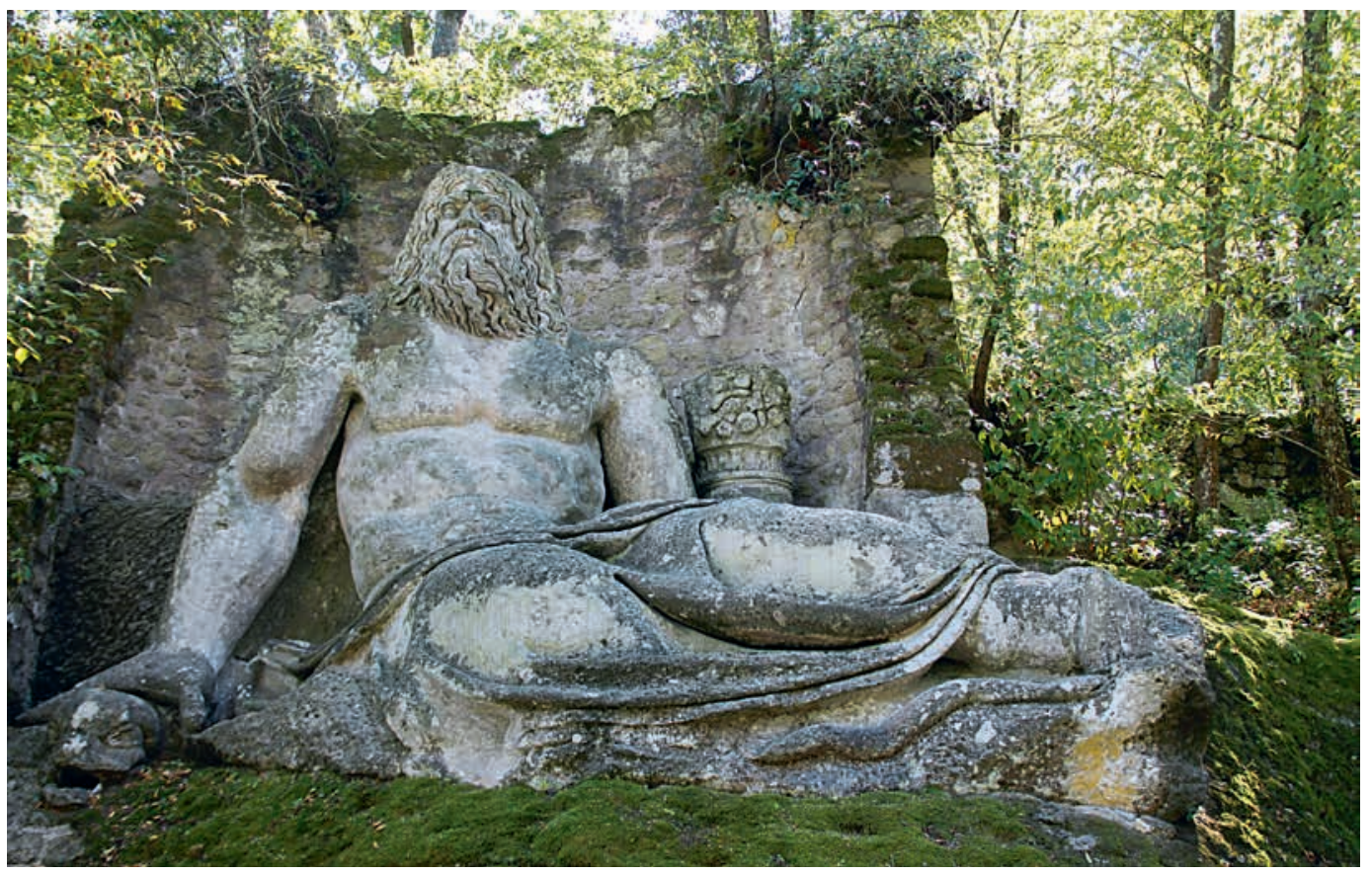

Abb. 13: Neptun. (Foto: P. Schubert)

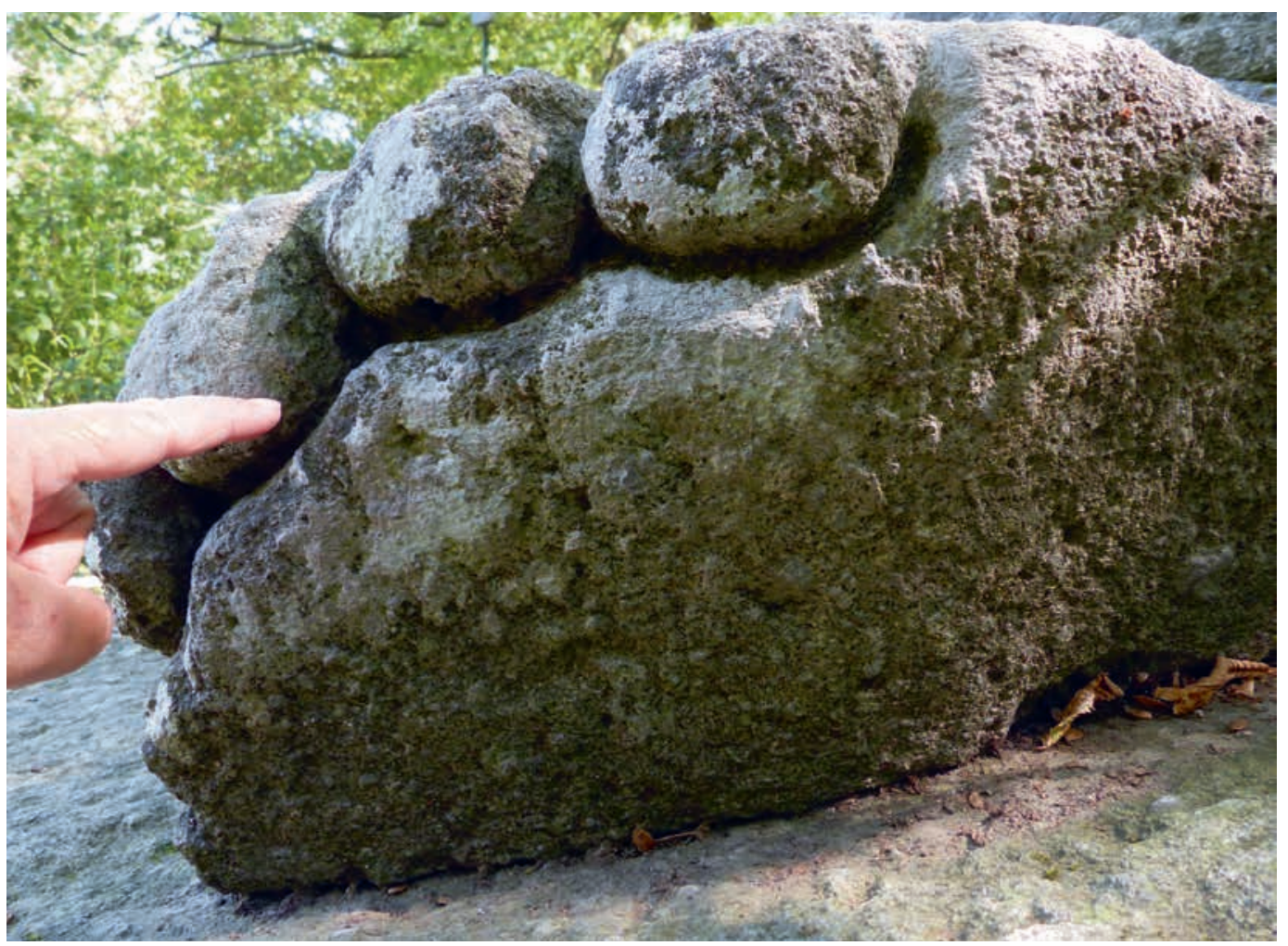

Abb. 14: Wem gehört denn dieser Riesenfuß? (Foto: H. Steinecke) 


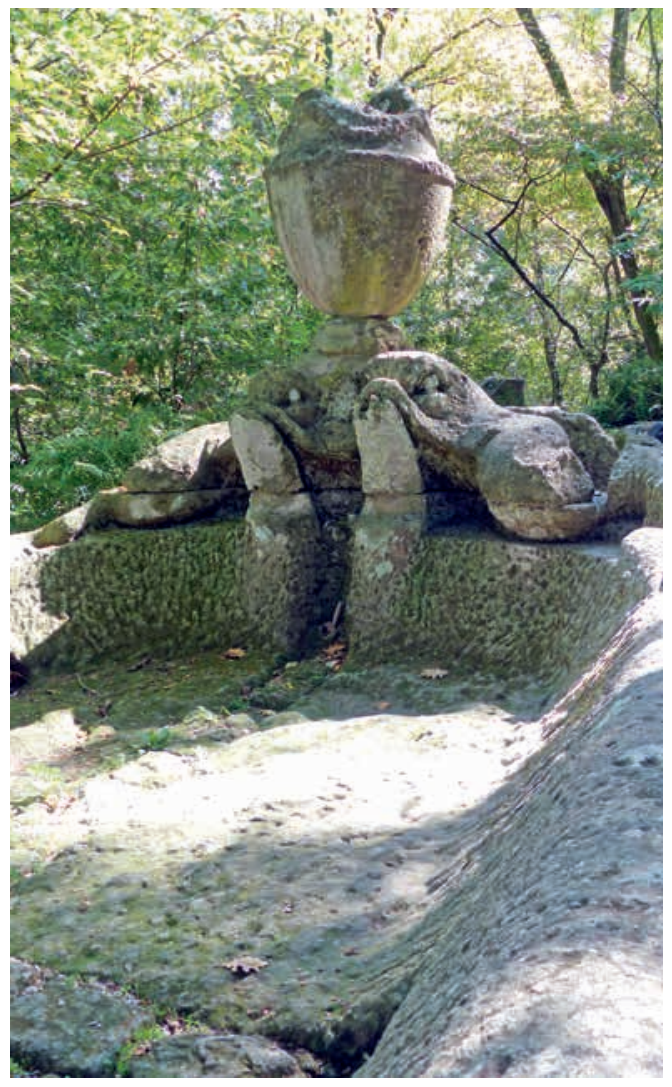

Abb. 15: Früher hat der Park auch zu einem Bad eingeladen. (Foto: H. Steinecke)

turmartige Haus neigt sich ähnlich wie der Schiefe Turm von Pisa. Beim Betreten des ersten Stockwerkes erfährt der Besucher eine Täuschung des Gleichgewichtssinnes und kann nicht mehr realisieren, ob er nun gerade oder schief steht oder ob Bäume senkrecht stehen oder nicht. Es stellt sich ein Schwindelgefühl ein. Es ist ein fantastischer Effekt, der letztendlich mit einfachen Mitteln schon vor über vier Jahrhunderten genutzt wurde, um die Besucher des Parks zu irritieren. Vielleicht hierzu besonders passend ist folgende Inschrift: „Du, der Du Stück für Stück mit Verstand hier herein kommst, sage mir hinterher, ob so viele Wunder aus Täuschungsabsicht oder um der Kunst willen gemacht worden sind“. Der aus der realen Welt kommende Besucher soll in der Casa Pendente wie auch in anderen Bereichen des Parks verunsichert werden und dabei gleichzeitig die Realität angezweifelt werden.
Der Besuch des Parkes Sacro Bosco ist äußerst lohnenswert, sowohl für diejenigen, die sich in eine geheimnisvolle Märchenwelt entführen lassen und einfach nur staunen wollen, sondern auch für an Kunst- und Gartengeschichte Interessierte. In etwa zwei Stunden hat man das Wichtigste im „Schnelldurchlauf" gesehen, so dass sich der Besuch auch selbst dann lohnt, wenn man auf der Durchreise, z. B. auf dem Weg von oder nach Rom ist und nur begrenzt Zeit zur Verfügung steht.

\section{Weiterführende Literatur und Internetseiten}

\section{Literatur}

De Filippis, Garcon, C. \& Greggio, S. 2014: Bomarzo: Le bois sacré/Il Sacro Bosco/The sacred Grove. - Des jardins d'exception. - Paris.

Hofmann, G. 2003: Bolsenasee und Umgebung. Reise- und Kulturführer. - Frankfurt am Main.

\section{Internetseiten}

https://science.orf.at/stories/2846039/

https://de.wikipedia.org/wiki/Sacro_Bosco

http://www.humboldtgesellschaft.de/inhalt.php?name=bomarzo

\section{Anschriften der Autorin und des Autors}

Dr. Hilke Steinecke, Palmengarten Frankfurt, Siesmayerstraße 61, 60323 Frankfurt, E-Mail: hilke.steinecke@ stadt-frankfurt.de

Dr. Peter Schubert, Universität Mainz, FB Biologie, Gresemundweg 2, 55128 Mainz, E-Mail: schubepe@unimainz.de 